ANNALES

POLONICI MATHEMATICI

LXXVIII.1 (2002)

\title{
Nonexistence results for the Cauchy problem of some systems of hyperbolic equations
}

\author{
by Mokhtar Kirane (Amiens) and \\ SAlim Messaoudi (Dhahran)
}

Abstract. We consider the systems of hyperbolic equations

$$
\begin{aligned}
& \begin{cases}u_{t t}=\Delta(a(t, x) u)+\Delta(b(t, x) v)+h(t, x)|v|^{p}, & t>0, x \in \mathbb{R}^{N}, \\
v_{t t}=\Delta(c(t, x) v)+k(t, x)|u|^{q}, & t>0, x \in \mathbb{R}^{N},\end{cases} \\
& \begin{cases}u_{t t}=\Delta(a(t, x) u)+h(t, x)|v|^{p}, & t>0, x \in \mathbb{R}^{N}, \\
v_{t t}=\Delta(c(t, x) v)+l(t, x)|v|^{m}+k(t, x)|u|^{q}, & t>0, x \in \mathbb{R}^{N},\end{cases} \\
& \begin{cases}u_{t t}=\Delta(a(t, x) u)+\Delta(b(t, x) v)+h(t, x)|u|^{p}, & t>0, x \in \mathbb{R}^{N}, \\
v_{t t}=\Delta(c(t, x) v)+k(t, x)|v|^{q}, & t>0, x \in \mathbb{R}^{N},\end{cases}
\end{aligned}
$$

in $(0, \infty) \times \mathbb{R}^{N}$ with $u(0, x)=u_{0}(x), v(0, x)=v_{0}(x), u_{t}(0, x)=u_{1}(x), v_{t}(0, x)=v_{1}(x)$. We show that, in each case, there exists a bound $\mathbf{B}$ on $N$ such that for $1 \leq N \leq \mathbf{B}$ solutions to the systems blow up in finite time.

1. Introduction. We consider the three nonlinear systems of hyperbolic equations (S1)-(S3) where $N, p, q, m \geq 1$ and $p q>1$, and $a(t, x), b(t, x)$, $c(t, x)$ are positive, bounded and measurable functions. The initial values are not necessarily compactly supported; however, they satisfy

Condition (H1).

$$
\int_{\mathbb{R}^{N}} u_{t}(0, x) \xi(0, x) d x \geq 0, \quad \int_{\mathbb{R}^{N}} v_{t}(0, x) \xi(0, x) d x \geq 0
$$

where

$$
\xi(t, x)=\phi^{\lambda}\left(\frac{t^{2}+|x|^{2}}{R^{2}}\right), \quad R>0, \lambda \text { large }
$$

2000 Mathematics Subject Classification: 35L15, 35L70.

Key words and phrases: blow up, hyperbolic system, weak solution. 
and $\phi \in C^{2}\left(\mathbb{R} ; \mathbb{R}^{+}\right)$is such that

$$
\phi(r)= \begin{cases}1 & \text { for } r \leq 1, \\ 0 & \text { for } r \geq 2,\end{cases}
$$

$0 \leq \phi \leq 1$ and $r\left|\phi^{\prime}(r)\right| \leq C$ for any $r>0$.

Concerning the functions $h(t, x) \geq 0, k(t, x) \geq 0$ and $l(t, x) \geq 0$, we assume that they satisfy:

Condition (H2).

$$
\left\{\begin{array}{l}
h(\tau R, y R) \simeq C_{0} R^{\mu}, \\
k(\tau R, y R) \simeq C_{0} R^{\kappa}, \\
l(\tau R, y R) \simeq C_{0} R^{\lambda},
\end{array}\right.
$$

for $R$ large, and for any $(\tau, y)$ in a bounded subset of $\mathbb{R}^{+} \times \mathbb{R}^{N} ; C_{0}$ is a positive constant.

For example:

$$
h(t, x)=t^{\sigma_{1}}|x|^{\varrho_{1}}, \quad k(t, x)=t^{\sigma_{2}}|x|^{\varrho_{2}}, \quad l(t, x)=t^{\sigma_{3}}|x|^{\varrho_{3}} .
$$

Before discussing our results in detail, let us briefly recall some results related to the single equation

$$
\begin{gathered}
u_{t t}=\Delta u+|u|^{p}, \quad x \in \mathbb{R}^{N}, t>0, \\
u(0, x)=u_{0}(x), \quad u_{t}(0, x)=u_{1}(x), \quad x \in \mathbb{R}^{N} .
\end{gathered}
$$

(i) For $N=1$, if $1<p<\infty$, every nontrivial solution of (3) blows up in finite time.

(ii) For $N=2,3$, there exists a "critical index" $p_{\mathrm{c}}(N)$ such that if $1<p$ $\leq p_{\mathrm{c}}(N)$ then $(3)$ has no nontrivial global solutions, while it admits nontrivial global small solutions if $p>p_{\mathrm{c}}(N)$, where $2 p_{\mathrm{c}}(2)=3+\sqrt{17}$ and $p_{\mathrm{c}}(3)=1+\sqrt{2}$.

Statement (i) was established by Glassey [3], Kato [5], and Sideris [10]. In statement (ii) the subcritical case was proved by John [4] when $N=3$ and by Glassey [3] for $N=2,3$; the critical case was settled by Schaeffer [9] for $N=2,3$; and the supercritical case was proved by Glassey [3] when $N=2$ and by John [4] for $N=3$. A valuable review of results on blowing up solutions to evolution equations is presented in [6].

REMARK 1. The case of (S1) with $a(t, x)=c(t, x)=h(t, x)=k(t, x)=1$, $b=0$ was considered by Keng Deng [1], [2]. His method of proof is based on the explicit formula for the required solution in terms of the fundamental solution for the wave equation. His method of proof is inoperative in our situation.

Our method of proof has been introduced in [7] and [8]. 
2. Results. In this section, the results concerning the nonexistence of nontrivial global solutions to (S1)-(S3) are presented.

Definition 1. For $0<T \leq \infty$ and $u_{0}, u_{1}, v_{0}, v_{1} \in L_{\text {loc }}^{1}\left(\mathbb{R}^{N}\right)$, we say that $(u, v)$ is a weak solution of (S1) if $u, v$ are locally integrable functions on $\mathbb{R}^{+} \times \mathbb{R}^{N}, u \in L_{\text {loc }}^{q}\left(\mathbb{R}^{+} \times \mathbb{R}^{N}, k(t, x) d t d x\right):=\left\{u: \mathbb{R}^{+} \times \mathbb{R}^{N} \rightarrow \mathbb{R} \mid\right.$ for any compact $\left.K, \int_{K}|u|^{q} k(t, x) d t d x<\infty\right\}, v \in L_{\text {loc }}^{p}\left(\mathbb{R}^{+} \times \mathbb{R}^{N}, h(t, x) d t d x\right)$, and for all $0<S<T$,

$$
\begin{aligned}
\int_{0}^{S} \int_{\mathbb{R}^{N}}\left(h|v|^{p} \varphi-u \varphi_{t t}\right. & +a u \Delta \varphi+b v \Delta \varphi) d t d x \\
& -\int_{\mathbb{R}^{N}} u_{0}(x) \varphi_{t}(0, x) d x+\int_{\mathbb{R}^{N}} u_{1}(x) \varphi(0, x) d x=0,
\end{aligned}
$$

and

$$
\begin{aligned}
\int_{0}^{S} \int_{\mathbb{R}^{N}}\left(k|u|^{q} \varphi-v \varphi_{t t}\right. & +c v \Delta \varphi) d t d x \\
& -\int_{\mathbb{R}^{N}} v_{0}(x) \varphi_{t}(0, x) d x+\int_{\mathbb{R}^{N}} v_{1}(x) \varphi(0, x) d x=0
\end{aligned}
$$

for all $0 \leq \varphi \in C^{2}\left([0, S] \times \mathbb{R}^{N}\right)$, with $\varphi(S, x)=0$ on $\mathbb{R}^{N}$ and $\varphi=0$ for $t \in[0, S]$ and $|x| \rightarrow \infty$. If $T=\infty$, we say that $(u, v)$ is a global weak solution.

The definitions of the solutions to (S2) and (S3) are clear.

Theorem 1. Assume that

$$
N \leq \mathbf{B}:=\min \left(\frac{\mu+1+p(\kappa+q+2)}{p q-1}, \frac{\mu+p+1}{p-1}\right)
$$

and $(\mathrm{H} 1),(\mathrm{H} 2)$ are satisfied. Then the system (S1) admits no solutions defined on $\mathbb{R}^{+} \times \mathbb{R}^{N}$.

Theorem 2. Assume that $m>p$,

$$
N \leq \mathbf{B}:=\frac{1}{p q-1} \max (\mu+p(\kappa+2+q), \kappa+q(\mu+2+p)),
$$

and (H1), (H2) are satisfied. Then the system (S2) admits no solutions defined on $\mathbb{R}^{+} \times \mathbb{R}^{N}$.

TheOREM 3. Assume that

$$
N \leq \mathbf{B}:=\min \left\{\frac{p+1+\mu}{p-1}, \frac{q+1+\kappa}{q-1}\right\},
$$

and (H1), (H2) are satisfied. Then the system (S3) admits no solutions defined on $\mathbb{R}^{+} \times \mathbb{R}^{N}$. 
REMARK. The integrability assumption on $u_{1}, v_{1}$ can be relaxed and the sign condition replaced by

$$
0 \leq \limsup _{R \rightarrow \infty} \int_{\mathbb{R}^{N}} u_{1}(x) \phi\left(|x|^{2} / R^{2}\right) d x, \quad 0 \leq \limsup _{R \rightarrow \infty} \int_{\mathbb{R}^{N}} v_{1}(x) \phi\left(|x|^{2} / R^{2}\right) d x .
$$

For the proofs of the theorems concerning (S1) and (S3), the following lemma is used in a crucial manner.

Lemma. Assume that $\alpha, \beta, y \in[0, \infty)$ and $\sigma \in(0,1)$. Then

$$
y \leq \alpha+\beta y^{\sigma} \Rightarrow y \leq \frac{\alpha}{1-\sigma}+\beta^{1 /(1-\sigma)} .
$$

Proof. For all $\alpha \in(0, \infty)$ the equation

$$
x=\alpha+\beta x^{\sigma}
$$

has exactly one solution $x=x(\alpha) \in(0, \infty)$. Hence (7) defines a function

$$
x: \alpha \in(0, \infty) \mapsto x(\alpha) \in(0, \infty) .
$$

Differentiating (7) with respect to $\alpha$ yields after some calculations

$$
x^{\prime}(\alpha)=\frac{x(\alpha)}{(1-\sigma) x(\alpha)+\sigma \alpha},
$$

hence

$$
x^{\prime}(\alpha)<\frac{1}{1-\sigma} \quad \text { for all } \alpha \in(0, \infty) .
$$

Thus

$$
x(\alpha) \leq \alpha \max _{\alpha \geq 0} x^{\prime}(\alpha)+x(0)=\frac{\alpha}{1-\sigma}+\beta^{1 /(1-\sigma)} .
$$

Since $y \leq x(\alpha)$ this proves the assertion (6).

Proof of Theorem 1. Assume to the contrary that (S1) has a global solution $(u, v)$. If we take as a test function the function $\xi$ defined in (1), we obtain

$$
\int h|v|^{p} \xi \leq \int\left(u \xi_{t t}-a u \Delta \xi-b v \Delta \xi\right)
$$

in the above inequality and hereafter, $\int:=\int_{\operatorname{supp} \xi}$ ( $\operatorname{supp}$ stands for support). Writing

$$
\begin{aligned}
\int|u| \cdot\left|\xi_{t t}\right| & =\int|u|(|\xi| k)^{1 / q}(|\xi| k)^{-1 / q}\left|\xi_{t t}\right|, \\
\int a|u| \cdot|\Delta \xi| & =\int a|u|(|\xi| k)^{1 / q}(|\xi| k)^{-1 / q}|\Delta \xi|, \\
\int b|v| \cdot|\Delta \xi| & =\int b|v|(|\xi| h)^{1 / p}(|\xi| h)^{-1 / p}|\Delta \xi|,
\end{aligned}
$$

and using the Hölder inequality, we obtain 
(9)

$$
\begin{aligned}
\int h|v|^{p} \xi d x \leq & \left(\int k|u|^{q} \xi\right)^{1 / q}\left\{\left(\int k^{-q^{\prime} / q} \xi^{-q^{\prime} / q}\left|\xi_{t t}\right|^{q^{\prime}}\right)^{1 / q^{\prime}}\right. \\
& \left.+\|a\|_{\infty}\left(\int k^{-q^{\prime} / q} \xi^{-q^{\prime} / q}|\Delta \xi|^{q^{\prime}}\right)^{1 / q^{\prime}}\right\} \\
& +\|b\|_{\infty}\left(\int h|v|^{p} \xi\right)^{1 / p}\left(\int h^{-p^{\prime} / p} \xi^{-p^{\prime} / p}|\Delta \xi|^{p^{\prime}}\right)^{1 / p^{\prime}}
\end{aligned}
$$

where $1 / p+1 / p^{\prime}=1 / q+1 / q^{\prime}=1,\|a\|_{\infty}:=\sup _{t, x}|a(t, x)|$, because of (H1), and the choice of the test function which ensures

$$
\int_{\mathbb{R}^{N}} u_{0}(x) \xi_{t}(0, x) d x=0
$$

since

$$
\xi_{t}=2 \lambda t R^{-2} \phi^{\prime}\left(\frac{t^{2}+|x|^{2}}{R^{2}}\right) \phi^{\lambda-1}\left(\frac{t^{2}+|x|^{2}}{R^{2}}\right) .
$$

Observe that for $\lambda \gg 1$, we have

$$
\begin{array}{ll}
\int k^{-q^{\prime} / q} \xi^{-q^{\prime} / q}\left|\xi_{t t}\right|^{q^{\prime}}<\infty, & \int k^{-q^{\prime} / q} \xi^{-q^{\prime} / q}|\Delta \xi|^{q^{\prime}}<\infty, \\
\int h^{-p^{\prime} / p} \xi^{-p^{\prime} / p}\left|\xi_{t t}\right|^{p^{\prime}}<\infty, & \int h^{-p^{\prime} / p} \xi^{-p^{\prime} / p}|\Delta \xi|^{p^{\prime}}<\infty .
\end{array}
$$

For simplicity we write (9) in the form

$$
\int h|v|^{p} \xi d x \leq\left(\int k|u|^{q} \xi\right)^{1 / q} \mathcal{A}_{a, k, q}+\mathcal{B}\left(\int h|v|^{p} \xi\right)^{1 / p}
$$

with a clear meaning of $\mathcal{A}_{a, k, q}$ and $\mathcal{B}$. Similarly, we have

$$
\int k|u|^{q} \xi \leq\left(\int h|v|^{p} \xi\right)^{1 / p} \mathcal{A}_{c, h, p} .
$$

Estimates (11) and (12) then give

$$
\int h|v|^{p} \xi \leq \mathcal{A}_{c, h, p}^{1 / q} \mathcal{A}_{a, k, q}\left(\int h|v|^{p} \xi\right)^{1 /(p q)}+\mathcal{B}\left(\int h|v|^{p} \xi\right)^{1 / p} .
$$

Now, estimate (13) can be arranged into

$$
\left(\int h|v|^{p} \xi\right)^{(p q-1) /(p q)} \leq \mathcal{A}_{c, h, p}^{1 / q} \mathcal{A}_{a, k, q}+\mathcal{B}\left(\int h|v|^{p} \xi\right)^{(q-1) /(p q)} .
$$

By setting

$$
\mathcal{X}:=\left(\int h|v|^{p} \xi\right)^{(p q-1) /(p q)},
$$

the latter inequality becomes

$$
\mathcal{X} \leq A+\mathcal{B} \mathcal{X}^{\sigma},
$$

where $0<\sigma:=(q-1) /(p q-1)<1$, and $A:=\mathcal{A}_{c, h, p}^{1 / q} \mathcal{A}_{a, k, q}$.

Now, using the Lemma, we obtain

$$
\mathcal{X} \leq \frac{A}{1-\sigma}+\mathcal{B}^{1 /(1-\sigma)} .
$$


At this stage, we introduce the scaled variables

$$
\tau:=R^{-1} t, \quad y:=R^{-1} x .
$$

We then obtain

$$
\begin{gathered}
d t d x=R^{1+N} d \tau d y, \quad \xi_{t}=R^{-1} \xi_{\tau}, \quad \Delta_{x} \xi=R^{-1} \Delta_{y} \xi \\
\mathcal{A}_{a, k, q} \leq C R^{-\kappa / q-1+N-1 / q-N / q}, \quad \mathcal{A}_{c, h, p} \leq C R^{-\mu / p-1+N-1 / p-N / p} .
\end{gathered}
$$

So

$$
A \leq C R^{s_{1}}, \quad \mathcal{B} \leq C R^{s_{2}},
$$

for some constant $C$ that may change from line to line, and where

$$
\begin{aligned}
& s_{1}:=\frac{1}{q}\left(-\frac{\mu}{p}-1+N-\frac{1}{p}-\frac{N}{p}\right)-\frac{\kappa}{q}-1+N-\frac{1}{q}-\frac{N}{q}, \\
& s_{2}:=-\frac{\mu}{p}-1+N-\frac{1}{p}-\frac{N}{p} .
\end{aligned}
$$

Accordingly

$$
\mathcal{X} \leq \frac{C}{1-\sigma} R^{s_{1}}+C R^{s_{2} /(1-\sigma)} .
$$

Now, we require $s_{1} \leq 0, s_{2} \leq 0$, which implies

$$
N \leq \min \left(\frac{\mu+1+p(\kappa+q+2)}{p q-1}, \frac{\mu+p+1}{p-1}\right) .
$$

We have two cases:

(i) $\max \left(s_{1}, s_{2}\right)<0$; in this case, when $R \rightarrow \infty$ in (15), the right-hand side will go to zero and hence $\int h|v|^{p}=0$, so that $v=0$, and $u=0$ by (12).

(ii) If $\max \left(s_{1}, s_{2}\right)=0$, say $s_{1}=0$, then from (15), if $\mathcal{D}:=\{(t, x) \mid$ $\left.R^{2} \leq t^{2}+|x|^{2} \leq 2 R^{2}\right\}$, we have $\int h|v|^{p} \leq C<\infty$ and $\operatorname{solim}_{R \rightarrow \infty} \int_{\mathcal{D}} h|v|^{p}=0$. We then use

$$
\int k|u|^{q} \xi \leq\left(\int_{\mathcal{D}} h|v|^{p} \xi\right)^{1 / p} \mathcal{A}_{c, h, p}
$$

and let $R \rightarrow \infty$ to obtain $\int k|u|^{q}=0$, and therefore $u=0$, which in turn implies $v=0$ via (9).

Proof of Theorem 2. The proof is similar to the one of Theorem 1. We have

$$
\int h|v|^{p} \xi \leq\left(\int k|u|^{q} \xi\right)^{1 / q} \mathcal{A}_{a, k, q}
$$

Also,

$$
\int l|v|^{m} \xi+\int k|u|^{q} \xi \leq\left(\int h|v|^{p} \xi\right)^{1 / p} \mathcal{A}_{c, h, p} .
$$

For the rest of the proof, we keep only the estimate

$$
\int k|u|^{q} \xi \leq\left(\int h|v|^{p} \xi\right)^{1 / p} \mathcal{A}_{c, h, p} .
$$


So

$$
\int k|u|^{q} \xi \leq\left(\int h|v|^{p} \xi\right)^{1 /(p q)} \mathcal{A}_{c, h, p}^{1 / q}
$$

Using (19) in (16), we obtain

$$
\int h|v|^{p} \xi \leq\left(\int h|v|^{p} \xi\right)^{1 /(p q)} \mathcal{A}_{c, h, p}^{1 / q} \mathcal{A}_{a, k, q},
$$

or

$$
\left(\int h|v|^{p} \xi\right)^{(p q-1) /(p q)} \leq \mathcal{A}_{c, h, p}^{1 / q} \mathcal{A}_{a, k, q} .
$$

Using the scaling, we obtain

$$
\mathcal{A}_{c, h, p} \leq C R^{-\frac{\mu}{p q}-\frac{p}{p q}+N \frac{p-1}{p q}-\frac{1}{p q}},
$$

so

$$
\mathcal{A}_{c, h, p}^{1 / q} \mathcal{A}_{a, k, q} \leq C R^{s}
$$

with

$$
s:=\frac{1}{p q}(-\mu-p-1-N(p-1))+\frac{1}{q}(-\kappa-q+N(q-1)-1) .
$$

Now, we require $s \leq 0$, and therefore

$$
N \leq \frac{\mu+p(\kappa+2+q)}{p q-1} .
$$

We could also start from the inequality

$$
\int k|u|^{q} \xi \leq\left(\int k|u|^{q} \xi\right)^{1 /(p q)} \mathcal{A}_{a, k, q} \frac{1}{p} \mathcal{A}_{c, h, p},
$$

which implies

$$
\left(\int k|u|^{q} \xi\right)^{(p q-1) /(p q)} \leq \mathcal{A}_{a, k, q} \frac{1}{p} \mathcal{A}_{c, h, p}
$$

The latter estimate analyzed through the scaling gives

$$
N \leq \frac{\kappa+q(\mu+2+p)}{p q-1} .
$$

So,

$$
N \leq \frac{1}{p q-1} \max (\mu+p(\kappa+2+q), \kappa+q(\mu+2+p)) .
$$

An analogous analysis as before leads to the result of Theorem 2.

Proof of Theorem 3. Here again, we present only the relevant estimates. We have

$$
\begin{aligned}
\mathcal{Y}:=\int k|v|^{q} \xi \leq & \left(\int k|v|^{q} \xi\right)^{1 / q}\left\{\left(\int k^{-q^{\prime} / q} \xi^{-q^{\prime} / q}\left|\xi_{t t}\right|^{q^{\prime}}\right)^{1 / q^{\prime}}\right. \\
& \left.+\|c\|_{\infty}\left(\int k^{-q^{\prime} / q} \xi^{-q^{\prime} / q}|\Delta \xi|^{q^{\prime}}\right)^{1 / q^{\prime}}\right\}
\end{aligned}
$$


or

$$
\begin{aligned}
\left(\int k|v|^{q} \xi\right)^{(q-1) / q} \leq & \left(\int k^{-q^{\prime} / q} \xi^{-q^{\prime} / q}\left|\xi_{t t}\right|^{q^{\prime}}\right)^{1 / q^{\prime}} \\
& +C\left(\int k^{-q^{\prime} / q} \xi^{-q^{\prime} / q}|\Delta \xi|^{q^{\prime}}\right)^{1 / q^{\prime}}
\end{aligned}
$$

with $1 / q+1 / q^{\prime}=1$.

We also have

$$
\int h|u|^{p} \xi \leq \int\left(\|a\|_{\infty}|u| \cdot|\Delta \xi|+\|b\|_{\infty}|v| \cdot|\Delta \xi|+|u| \cdot\left|\xi_{t t}\right|\right) .
$$

Using the Hölder inequality, we obtain

$$
\mathcal{X}:=\int h|u|^{p} \xi \leq\left(\int h|u|^{p} \xi\right)^{1 / p} \mathcal{D}_{h, p}+\left(\int k|v|^{q} \xi\right)^{1 / q} \mathcal{L}
$$

where

$$
\begin{aligned}
\mathcal{D}_{h, p} & :=\left(\int h^{-p^{\prime} / p} \xi^{-p^{\prime} / p}\left|\xi_{t t}\right|^{p^{\prime}}\right)^{1 / p^{\prime}}+\|a\|_{\infty}\left(\int h^{-p^{\prime} / p} \xi^{-p^{\prime} / p}|\Delta \xi|^{p^{\prime}}\right)^{1 / p^{\prime}} \\
\mathcal{L} & :=\left(\int k^{-q^{\prime} / q} \xi^{-q^{\prime} / q}|\Delta \xi|^{q^{\prime}}\right)^{1 / q^{\prime}}\|b\|_{\infty}
\end{aligned}
$$

So

$$
\mathcal{X} \leq \mathcal{X}^{1 / p} \mathcal{D}_{h, p}+\mathcal{Y}^{1 / q} \mathcal{L}
$$

From $(20), \mathcal{Y}^{(q-1) / q} \leq \mathcal{D}_{k, q}$, so

$$
\mathcal{X} \leq \mathcal{X}^{1 / p} \mathcal{D}_{h, p}+\mathcal{L} \mathcal{D}_{k, q}^{1 /(q-1)}
$$

and by the Lemma,

$$
\mathcal{X} \leq \frac{p \mathcal{L} \mathcal{D}_{k, q}^{1 /(q-1)}}{p-1}+\mathcal{D}_{h, p}^{p /(p-1)} .
$$

Using the scaling, we obtain

$$
\mathcal{L} \leq C R^{-\kappa / q-1+N-1 / q-N / q}, \quad \mathcal{D}_{k, q} \leq C R^{-\mu / p-1+N-1 / p-N / p} .
$$

So

$$
\mathcal{L} \mathcal{D}_{k, q}^{1 /(q-1)} \leq C R^{-\kappa / q-1+N-1 / q-N / q}
$$

An analysis of the inequalities leads to

$$
N \leq \min \left\{\frac{p+1+\mu}{p-1}, \frac{q+1+\kappa}{q-1}\right\},
$$

which is the constraint required in the theorem.

The rest of the proof is analogous to that of Theorem 1 , hence is omitted. 


\section{References}

[1] K. Deng, Blow-up of solutions of some nonlinear hyperbolic systems, Rocky Mountain J. Math. 29 (1999), 807-820.

[2] - Nonexistence of global solutions of a nonlinear hyperbolic system, Trans. Amer. Math. Soc. 349 (1997), 1685-1696.

[3] R. T. Glassey, Finite-time blow-up for solutions of nonlinear wave equations, Math. Z. 177 (1981), 323-340.

[4] F. John, Blow-up of solutions of nonlinear wave equations in three space dimensions, Manuscripta Math. 28 (1979), 235-268.

[5] T. Kato, Blow-up of solutions of some nonlinear hyperbolic equations, Comm. Pure Appl. Math. 33 (1980), 501-505.

[6] H. A. Levine, The role of critical exponents in blowup theorems, SIAM Rev. 32 (1990), 262-288.

[7] E. Mitidieri and S. I. Pokhozhaev, Absence of positive solutions for systems of quasilinear elliptic equations and inequalities in $R^{N}$, Dokl. Akad. Nauk 366 (1999), 13-17 (in Russian).

[8] S. I. Pokhozhaev and L. Véron, Blow-up results for nonlinear hyperbolic inequalities, preprint, Univ. de Tours, 1999.

[9] J. Schaeffer, The equation $u_{t t}-\Delta u=|u|^{p}$ for the critical value of $p$, Proc. Roy. Soc. Edinburgh Sect. A 101 (1985), 31-44.

[10] T. C. Sideris, Nonexistence of global solutions to semilinear wave equations in high dimensions, J. Differential Equations 52 (1984), 378-406.

Université de Picardie Jules Verne

Faculté de Mathématiques

et d'Informatique

LAMFA UPRES

A 6119, 33 rue Saint Leu

80039 Amiens Cedex 1, France

E-mail: Mokhtar.Kirane@u-picardie.fr

Current address:

Laboratoire de Mathématiques

Pôle Sciences et Technologies

Université de La Rochelle

Avenue Michel Crépeau

17042 La Rochelle Cedex, France

E-mail: mokhtar.kirane@univ-lr.fr
Department of Mathematical Sciences

King Fahd University of Petroleum and Minerals Dhahran 31261, Saudi Arabia E-mail: messaoud@kfupm.edu.sa 